DOI: http://dx.doi.org/10.12957/demetra.2015.8825

\title{
Perfil antropométrico, cardiorrespiratório e bioquímico de adolescentes estudantes de um instituto federal do Rio Grande do Sul
}

\section{Anthropometric, cardiorespiratory and biochemical profile of adolescent students of a federal institute in Rio Grande do Sul state, Brazil}

\author{
Carlise Felki Prevedellor \\ Valeriano Antonio Corbellini² \\ Miriam Beatrís Reckziegel ${ }^{3}$ \\ Renato Xavier Coutinho ${ }^{4}$ \\ Nelci José Donadel ${ }^{4}$ \\ Hildegard Hedwig Pohl ${ }^{3}$ \\ ${ }^{1}$ Instituto Federal Farroupilha, Setor de Nutrição. \\ São Vicente do Sul-RS, Brasil. \\ ${ }^{2}$ Universidade de Santa Cruz do Sul, Programa \\ de Pós-Graduação em Promoção da Saúde, \\ Departamento de Química e Física. Santa Cruz do \\ Sul-RS, Brasil. \\ ${ }^{3}$ Universidade de Santa Cruz do Sul, Programa \\ de Pós-Graduação em Promoção da Saúde, \\ Departamento de Educação Física e Saúde. Santa \\ Cruz do Sul-RS, Brasil. \\ ${ }^{4}$ Instituto Federal Farroupilha, Setor de Educação \\ Física. São Vicente do Sul-RS, Brasil. \\ Correspondência / Correspondence \\ Renato Xavier Coutinho \\ renatocoutinho@msn.com
}

\section{Resumo}

O estudo teve por objetivo traçar o perfil antropométrico, cardiorrespiratório e bioquímico de adolescentes estudantes de um instituto federal do Rio Grande do Sul. Participaram 63 alunos do primeiro ano do ensino médio integrado, sendo avaliados o índice de massa corpórea, a circunferência da cintura, o percentual de gordura, o consumo máximo de oxigênio, glicose, colesterol total e triglicerídeos. Observaramse diferenças significativas entre os sexos no percentual de gordura e consumo máximo de oxigênio. Também se verificou importante porcentagem da população com excesso de peso, excesso de gordura e valores de colesterol total acima do recomendado para essa faixa etária. Assim, evidenciou-se a importância de que medidas de prevenção do aumento ou manutenção de peso considerado saudável sejam implantadas nessa população, como instrumentos para promover a saúde e qualidade de vida desses alunos.

Palavras-chave: Adolescente. Estudante. Perfil de Saúde. Ganho de peso.

\section{Abstract}

The study aimed to trace the anthropometric, cardiorespiratory and biochemical profile of adolescent students of a federal institute in Rio Grande do Sul state, Brazil. Participants were 63 freshman students of the integrated high school, and were assessed body 
mass index, waist circumference, body fat percentage, maximum oxygen consumption, glucose, total cholesterol and triglycerides. There were significant differences between the sexes in the percentage of fat and maximal oxygen uptake. Also there was significant percentage of the population overweight, excess fat and total cholesterol levels above recommended for this age group. Thus, these results evidence the importance of developing prevention actions against the weight gain, or for the maintaining of healthy weight in this population, in order to promote health and quality of life for these students.

Key words: Adolescent. Student. Health Profile. Weight Gain.

\section{Introdução}

A adolescência, de acordo com a fisiologia, é definida como a soma dos processos biológicos que visam atingir a capacidade reprodutiva. Mecanismos hormonais que regulam esta fase acarretam aumento significativo nas dimensões e no peso. Observam-se também, nesta fase, alterações na composição corporal, no crescimento somático e no surgimento dos caracteres sexuais, que se iniciam em idades diferentes. Essas diferenças estão relacionadas a sexo, raça e características ambientais e culturais em que o adolescente está inserido. A adolescência não é apenas uma fase de evolução do ponto de vista hormonal e fisiológico, mas também de desenvolvimento psíquico, definições de identidade e hábitos para a vida futura do indivíduo., ${ }^{1,2}$

O termo "adolescência" vem do verbo latino "adolescere" ( $a d=$ para e olescere $=$ crescer), fase de desenvolvimento da identidade adulta, de transição para a maturidade, na qual são vividas descobertas e mudanças corporais e psicológicas, com o desenvolvimento físico precedendo o psicológico. É caracterizada por intenso crescimento e desenvolvimento, que se manifesta por marcantes transformações anatômicas, fisiológicas, psicológicas e sociais. ${ }^{3-6}$

Na adolescência, o indivíduo experimenta os melhores índices de saúde, os quais podem ser mantidos e/ou melhorados, dependendo da percepção e valorização dos mesmos. Portanto, o cenário de transição nutricional e epidemiológica da adolescência tem sido apontado como período estratégico de prevenção de doenças e promoção de saúde, influenciando diretamente na qualidade de vida dessa população. A deteç̧ão precoce de agravos, o tratamento adequado e a reabilitação devem se fundamentar na integralidade das ações e, ao mesmo tempo, apresentar estratégias articuladas de ações preventivas e curativas. Abordar a saúde do adolescente 
implica pensar nas mudanças biológicas, sociais, comportamentais e nos diversos modos de viver a adolescência, que culminarão na concepção dos adultos do futuro. Tratar o adolescente de forma adequada significa prevenir gastos e agravos ao futuro do país. ${ }^{7,8}$

O sobrepeso e a obesidade na infância já podem ser considerados uma epidemia, que engloba os países desenvolvidos e os países em desenvolvimento, atingindo igualmente meninos e meninas, e se associa a problemas de saúde mais frequentes e a custos cada vez mais elevados. A obesidade na adolescência pode resultar em aumento na pressão arterial, dislipidemias, diabetes, além de distúrbios psicológicos, emocionais e de relacionamento, todos estes aspectos culminam em maior morbimortalidade na idade adulta, especialmente pelo desenvolvimento de doenças cardiovasculares. ${ }^{9-11}$

A Organização Mundial da Saúde determina que medidas efetivas para o controle da obesidade devem incluir a abordagem preventiva e de promoção da saúde na infância e adolescência, a fim de evitar sua permanência ou agravamento na idade adulta. Para isso, torna-se necessário identificar aqueles indivíduos com maior risco de desenvolver complicações decorrentes do excesso de peso e adotar medidas para protegê-los..$^{12,13}$

Nesse contexto, pretende-se traçar o perfil antropométrico, cardiorrespiratório e bioquímico de adolescentes estudantes de um instituto federal localizando no Estado do Rio Grande do Sul.

\section{Método}

Os sujeitos do presente estudo, de caráter transversal, foram 63 alunos do primeiro ano dos cursos técnicos integrados ao segundo grau matriculados em 2012, no Instituto Federal Farroupilha Campus São Vicente do Sul (IFF-SVS), que aderiram, voluntariamente, ao estudo mediante a entrega do Termo de Consentimento Livre e Esclarecido (TCLE), assinado pelos pais ou responsáveis. Os cursos participantes foram os de Manutenção e Suporte a Informática e Agropecuária.

A amostra foi composta por 19 estudantes do sexo feminino (30,2\%) e 44 do sexo masculino (69,8\%). Os critérios de inclusão adotados foram: estar matriculado pela primeira vez no primeiro ano dos cursos técnicos integrados deste instituto federal e apresentar o TCLE assinado. Os critérios de exclusão estipulados foram: idade superior a 18 anos e patologias que possam interferir na avaliação nutricional, cardiorrespiratória e bioquímica, como obesidade mórbida e/ou problemas respiratórios. Nenhum estudante que retornou com o TCLE assinado possuía idade superior a 18 anos e possuía patologias que pudessem o excluir do estudo, portanto nesta fase não foram excluídos alunos. 
Dos 161 TCLE entregues, 67 foram devolvidos assinados, dos quais dois estudantes não compareceram no dia da coleta de dados; de um não foi possível a retirada de sangue durante a coleta e uma aluna se recusou a realizar o teste de Cooper. Desta forma, quatro alunos foram excluídos do estudo. Em março foi realizada aferição de peso, estatura e circunferência da cintura pela pesquisadora. No mês de abril, foi efetuada a coleta de sangue, com os alunos em jejum de 12 horas. Após a coleta, os alunos tomaram café da manhã, aguardaram no mínimo 30 minutos e após foram encaminhados para a aferição das dobras cutâneas e realização do teste de Cooper.

Para a avaliação nutricional, foram estudadas as variáveis antropométricas índice de massa corpórea $\left(\mathrm{IMC}=\mathrm{kg} / \mathrm{m}^{2}\right)$, circunferência da cintura $(\mathrm{CC})$ e percentual de gordura $(\% \mathrm{G})$, observandose, na avaliação, os critérios e recomendações preconizadas por Heyward (2004). ${ }^{14}$ Os valores do IMC foram identificados pelas Curvas WHO, 2007/NCHS, $1977,{ }^{15}$ observando-se sexo e idade, e classificados conforme preconiza a OMS. ${ }^{16}$ Para tanto, foram definidas três categorias: baixo-peso (percentil $>5$ ), eutrofia (percentis entre $\geq 5$ a 85) e excesso de peso (percentil 85).

Os valores da circunferência da cintura (CC) foram classificados pela tabela de percentis para crianças e adolescentes de Taylor et al., ${ }^{17}$ sendo considerado excesso de peso os valores acima do percentil 80. Já o percentual de gordura $(\% \mathrm{G})$ foi determinado pela espessura das dobras cutâneas triciptal (DCTR) e subescapular (DCSE); a partir da soma destas dobras, classificou-se como excesso de peso o percentil $>90$ de acordo com a metodologia de Frisancho, ${ }^{18}$ enquanto para a estimativa do $\% \mathrm{G}$ utilizou-se a metodologia de Lohman et al. ${ }^{19}$

A capacidade cardiorrespiratória, avaliada pelo consumo máximo de oxigênio $\left(\mathrm{VO}_{2 \operatorname{má}}\right)$, foi aferida através da aplicação do teste de Cooper, na pista de atletismo da instituição, constando de corrida contínua, em pista livre de 500 metros, durante o período de 12 minutos, observandose, na aplicação do teste, os critérios propostos por Cooper. ${ }^{20} \mathrm{O} \mathrm{VO}_{2 \text { máx. }}$ foi calculado a partir da distância percorrida individualmente, através da fórmula: $\left[\mathrm{VO}_{2 \text { máx. }}=(\mathrm{m}-504,9) / 44,73\right]$, em que "m" indica a distância em metros que cada indivíduo conseguiu alcançar. ${ }^{21,22}$ Essas medidas foram aferidas por avaliadores voluntários da UNISC, previamente treinados e habilitados.

Os exames bioquímicos glicose $(\mathrm{G})$, colesterol total (CT) e triglicerídeos (TG) foram prescritos pelo médico da instituição, sendo a coleta de sangue, para as avaliações bioquímicas, realizada por profissionais voluntários e habilitados da UNISC e uma técnica em enfermagem contratada. Antecedendo a coleta de sangue, os sujeitos foram orientados a manter jejum de 12 horas, como também não fazer exercícios físicos nas 12 horas que antecederam a coleta. Foram coletados cerca de $10 \mathrm{ml}$ de sangue da veia braquial; o sangue foi colocado em tubos com anticoagulante, para posterior centrifugação e obtenção de amostras de plasma para análise nos laboratórios da UNISC.

Segundo os padrões definidos e simplificados pela OMS e a Sociedade Brasileira de Diabetes, foram classificados como diabéticos (DM) os indivíduos que apresentaram glicemia de jejum $\geq$ a 
$126 \mathrm{mg} / \mathrm{dl}$; para os não diabéticos (GN) foram considerados normais valores < 100mg/dl e tolerância a glicose diminuída (TGD), os valores entre 100 e 126mg/dl. ${ }^{23}$ Para avaliação das dislipidemias, foram considerados os valores de referência lipídica propostos para a faixa etária de 2 a 19 anos da I Diretriz Brasileira de Prevenção da Aterosclerose na infância e na adolescência. Os valores considerados desejáveis de CT são aqueles menores de $150 \mathrm{mg} / \mathrm{dl}$ (CD); valores limítrofes, entre 150 e 169mg/dl (CL); e considerados com colesterol aumentado os que apresentaram níveis de CT acima de 170mg/dl (CA). Em relação aos triglicerídeos, foram considerados desejáveis valores $>100 \mathrm{mg} / \mathrm{dl}$ (TD); valores limítrofes, entre 100 e 129mg/dl (CL); e triglicerídeo aumentado (TA) os valores $\leq 130 \mathrm{mg} / \mathrm{dl} .^{24,25}$

As variáveis contínuas foram apresentadas através de média e desvio padrão. A tabulação dos dados foi feita em planilha eletrônica (Excel, Microsoft Office 2000) e estes analisados no Statistical Package for Social Sciences for Windows (SPSS - versão 20.0).

O projeto que deu origem ao estudo foi aprovado pelo Comitê de Ética em pesquisa da Universidade de Santa Cruz do Sul (UNISC), sob protocolo ${ }^{\circ}$ 2.960/11, e autorizado pelo diretor geral do IFF-SVS.

\section{Resultados}

Dos 63 adolescentes avaliados, 69,8\% eram do sexo masculino, a maioria no intervalo etário dos 14 aos 15 anos (14,6 \pm 0,789), todos ingressantes do primeiro ano do ensino médio do IFFSVS (tabela 1).

Tabela 1. Características demográficas dos estudantes do primeiro ano do ensino médio do IFF-SVS. Tabela 1. Características demográficas dos estudantes do primeiro ano do ensino médio do IFF-SVS. São Vicente do Sul-RS, 2012.

\begin{tabular}{lccc} 
& $\begin{array}{c}\text { Geral } \\
\%(\mathrm{n})\end{array}$ & $\begin{array}{c}\text { Feminino } \\
\%(\mathrm{n})\end{array}$ & $\begin{array}{c}\text { Masculino } \\
\%(\mathrm{n})\end{array}$ \\
\hline Amostra & $100(63)$ & $30,2(19)$ & $\mathbf{6 9 , 8} \mathbf{( 4 4 )}$ \\
Idade (anos) & $14,6 \pm 0,789$ & $14,5 \pm 0,697$ & $14,7 \pm 0,829$ \\
Faixa Etária & & & \\
$14-15$ anos & $\mathbf{8 4 , 2 ( 5 3 )}$ & $89,5(17)$ & $81,8(36)$ \\
$16-17$ anos & $15,8(10)$ & $10,5(2)$ & $18,2(8)$ \\
\hline
\end{tabular}

* Media \pm desvio padrão. 
A tabela 2 detalha as características antropométricas, cardiorrespiratória e bioquímicas dos estudantes, com dados expressos em média e desvio padrão, com significância dos dados calculados entre os grupos feminino e masculino. Os resultados de IMC e \%G apresentam valores mais elevados no sexo feminino, enquanto o $\mathrm{VO}_{2 \text { máx }}$ foi maior no sexo masculino. Foram ainda observadas diferenças significativas entre os sexos nas variáveis $\% \mathrm{G}$ e $\mathrm{VO}_{2 \text { máx }}$.

Tabela 2. Características antropométricas, cardiorrespiratória e bioquímicas dos estudantes do primeiro ano do ensino médio do IFF-SVS. São Vicente do Sul-RS, 2012.

\begin{tabular}{lccc}
\hline Variáveis & $\begin{array}{c}\text { Geral }(\mathrm{n}=63) \\
\overline{\mathrm{x}} \pm \mathrm{DP}\end{array}$ & $\begin{array}{c}\text { Feminino }(\mathrm{n}=19) \\
\overline{\mathrm{x}} \pm \mathrm{DP}\end{array}$ & $\begin{array}{c}\text { Masculino }(\mathrm{n}=44) \\
\overline{\mathrm{x}} \pm \mathrm{DP}\end{array}$ \\
\hline Antropométricas & & & \\
IMC & $21,3 \pm 3,4$ & $22,6 \pm 2,9$ & $20,9 \pm 3,6$ \\
CG & $74,9 \pm 9,1$ & $73,5 \pm 8,4$ & $75,5 \pm 9,5$ \\
\%G & $22,8 \pm 8,1$ & $28,6 \pm 5,6$ & $20,3 \pm 7,8$ \\
Cardiorrespiratória & & & \\
VO2máx. & $30,2 \pm 8,6$ & $21,2 \pm 4,0$ & $34,1 \pm 7,0$ \\
Bioquímicas & & & \\
Glicose & $88,0 \pm 7,8$ & $85,9 \pm 8,8$ & $88,9 \pm 7,2$ \\
Colesterol & $149,9 \pm 25,4$ & $158,5 \pm 24,2$ & $146,2 \pm 25,3$ \\
Triglicerídeos & $42,5 \pm 19,7$ & $49,3 \pm 19,8$ & $39,6 \pm 2,8$ \\
\hline
\end{tabular}

IMC - índice de massa corpórea; CC - circunferência da cintura; \% G - percentual de gordura.

Ao classificar as características antropométricas dos estudantes através de percentis (tabela 3), podemos perceber que a maioria dos estudantes foi classificada na categoria "eutrofia", com maior percentual de meninas eutróficas em todas as variáveis, não obstante a presença de excesso de peso em 31,6\% das meninas. Cabe ressaltar que, de acordo com a circunferência da cintura, $29,5 \%$ do sexo masculino apresentaram excesso de peso. 
Tabela 3. Características antropométricas dos estudantes do primeiro ano do ensino médio do IFF-SVS, classificadas de acordo com os percentis. São Vicente do Sul-RS, 2012.

\begin{tabular}{cccc}
\hline Variáveis & $\begin{array}{c}\text { Geral } \\
\%(\mathrm{n})\end{array}$ & $\begin{array}{c}\text { Feminino } \\
\%(\mathrm{n})\end{array}$ & $\begin{array}{c}\text { Masculino } \\
\%(\mathrm{n})\end{array}$ \\
\hline IMC BP & $9,5(6)$ & $0(0)$ & $13,6(6)$ \\
Eutrofia & $68,3(43)$ & $68,4(13)$ & $68,2(30)$ \\
EP & $22,2(14)$ & $31,6(6)$ & $18,2(8)$ \\
CC Eutrofia & $71,4(45)$ & & $70,5(31)$ \\
EP & $28,6(18)$ & $26,3(5)$ & $29,5(13)$ \\
\%G Eutrofia & $88,9(56)$ & & $86,4(38)$ \\
EP & $11,1(7)$ & $94,7(18)$ & $13,6(6)$ \\
\hline
\end{tabular}

IMC - índice de massa corpórea; CC - circunferência da cintura; \%G - percentual de gordura. BP - baixopeso; EP - excesso de peso.

Os resultados das características bioquímicas dos estudantes estão expressos na tabela 4 e demonstram que 97,3\% dos estudantes, de ambos os sexos, apresentaram valores de glicose dentro do recomendado pela OMS e a Sociedade Brasileira de Diabetes. Embora não havendo ocorrência de diabéticos, observou-se presença de 6,3\% dos estudantes, a maioria masculina, com tolerância a glicose diminuída. Em relação ao colesterol total, 50,8\% dos estudantes apresentaram-se dentro dos limites considerados saudáveis, e não obstante este resultado, 31,6\% do sexo feminino e 31,8\% do sexo masculino foram classificados com colesterol limítrofe (CL). Quanto aos triglicerídeos, todos os estudantes (100\%) apresentaram classificação desejável. 
Tabela 4. Características bioquímicas dos estudantes do primeiro ano do ensino médio do IFF-SVS, classificadas de acordo com os valores de referência para a faixa etária. São Vicente do Sul-RS, 2012.

\begin{tabular}{|c|c|c|c|c|}
\hline \multicolumn{2}{|l|}{ Variáveis } & $\begin{array}{l}\text { Geral } \\
\%(\mathrm{n})\end{array}$ & $\begin{array}{c}\text { Feminino } \\
\%(\mathrm{n})\end{array}$ & $\begin{array}{c}\text { Masculino } \\
\%(\mathrm{n})\end{array}$ \\
\hline \multirow[t]{3}{*}{ Glicose } & GN & $93,7(59)$ & 94,7 (18) & 93,2 (41) \\
\hline & TGD & $6,3(4)$ & $5,3(1)$ & $6,8(3)$ \\
\hline & $\mathrm{DM}$ & $0(0)$ & $0(0)$ & $0(0)$ \\
\hline \multirow[t]{3}{*}{ Colesterol } & CD & $50,8(32)$ & $42,1(8)$ & $54,5(24)$ \\
\hline & CL & $31,7(20)$ & $31,6(6)$ & $31,8(14)$ \\
\hline & CA & $17,5(11)$ & $26,3(5)$ & $13,6(6)$ \\
\hline \multirow[t]{3}{*}{ Triglicerídeos } & TD & $100(63)$ & $100(19)$ & $100(44)$ \\
\hline & TL & $0(0)$ & $0(0)$ & $0(0)$ \\
\hline & TA & $0(0)$ & $0(0)$ & $0(0)$ \\
\hline
\end{tabular}

GN - glicose normal; TGD - tolerância a glicose diminuída; DM - diabetes melito; CD - colesterol Desejável; CL - colesterol limítrofe; CA - colesterol aumentado; TD - triglicerídeos desejáveis TL - triglicerídeos limítrofes; triglicerídeos aumentados.

\section{Discussão}

Neste estudo, buscou-se traçar o perfil antropométrico, cardiorrespiratório e bioquímico de estudantes ingressantes no primeiro ano do segundo grau do IFF-SVS. Constatou-se importante porcentagem de sujeitos com excesso de peso, excesso de gordura e valores de colesterol total acima do recomendado para esta população. Esse fato preocupa, pela precocidade de manifestação do excesso de peso e suas consequências para a saúde.

Os índices de massa corpórea e percentual de gordura dos estudantes do presente estudo, considerando os desvios padrão, são semelhantes aos encontrados em estudo com escolares de Santa Cruz do Sul-RS, ${ }^{26}$ que demonstrou IMC de 20,73 $\pm 4,09$ no sexo feminino e 20,15 $\pm 3,74$ no

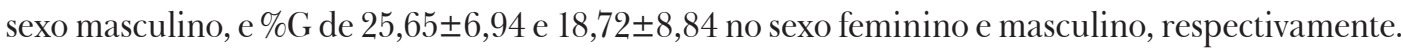
Devem-se considerar, no enanto, as diferenças de idade dos sujeitos entre os dois estudos. 
Resultados de $\mathrm{VO}_{2 \text { máx. }}$ mais elevado no sexo masculino, quando comparados com o sexo feminino, são mais frequentes na literatura. ${ }^{27}$ Fatores culturais para a prática de atividade física nos meninos, assim como maior propensão de desenvolvimento muscular nesse sexo, favorecem esse diagnóstico. Já em relação ao sexo feminino, a maior quantidade de gordura corporal pode favorecer estas diferenças, segundo estudo de Cotinguiba e Sergipe. ${ }^{27}$

Tal constatação converge com nosso estudo, uma vez que encontramos um \% maior nas meninas. Este fato pode ser explicado devido à tendência das adolescentes do sexo feminino a ganharem maior quantidade de massa gorda, em virtude do aumento dos níveis de estrogênio na puberdade, enquanto os adolescentes do sexo masculino têm aumento dos níveis de testosterona. Desta forma, o aumento da massa gorda refletirá em maior \%G.

Dos adolescentes estudados em ambos os sexos, $68,3 \%$ foram classificados como eutróficos, de acordo com seu IMC, porém o fato de 22,2\% da população estudada apresentar excesso de peso e 28,6\% apresentar excesso do gordura corporal é preocupante, visto que o excesso de peso e gordura corporal podem levar à obesidade, e mesmo nessa faixa etária, tal situação poderá estar relacionada a fatores de risco para doenças cardiovasculares, aumentando a morbimortalidade relacionada à obesidade no adulto. ${ }^{28,29}$

Em estudo com escolares de Itapetininga-SP, ${ }^{30}$ com idade de 2 a 19 anos, foram encontrados $22,5 \%$ de sobrepeso e obesidade (>P85), valores semelhantes ao presente estudo. Isso demonstra que o excesso de peso encontrado neste estudo com amostra de 63 estudantes do instituto federal está próximo da realidade de outras amostras de estudos realizados em nosso país.

Segundo a Pesquisa de Orçamentos Familiares (POF) 2008-2009, ${ }^{31}$ realizada nacionalmente com crianças e adolescentes de 10 a 19 anos, no sexo feminino, o excesso de peso passou de 7,6\%, na pesquisa de 1974-75, para 19,4\%, em 2008-09; e no sexo masculino, de 3,7\%, para 21,7\%. Portanto, os quantitativos de excesso de peso encontrados nesta pesquisa para o sexo feminino estão mais elevados, e no sexo masculino mais baixos, se compararmos com a POF 2008-2009.

Tendo em vista o número crescente de crianças e adolescentes que apresentam risco de doenças cardiovasculares, ${ }^{32}$ outro fato preocupante é a constatação de que 31,6\% dos sujeitos do sexo feminino e $31,8 \%$ do sexo masculino foram classificados com colesterol limítrofe (CL). Esses valores são superiores ao estudo com escolares realizado em Belém-PA, ${ }^{33}$ no qual o CL estava em $18 \%$ no sexo feminino e $16 \%$ no masculino.

Como se observa, os fatores desencadeantes da dislipidemia também estão sendo encontrados nas crianças e adolescentes. Atualmente, os fatores de risco cardiovasculares descritos tradicionalmente em adultos estão sendo estendidos à população pediátrica. ${ }^{34,35}$ A hiperlipidemia já é considerada fator importante para a ocorrência de aterogênese nas crianças e adolescentes, ${ }^{36}$ e a manutenção 
das frações lipídicas ao longo da vida adulta também já é descrita, visto que a maioria das crianças mantém os níveis de colesterol até a vida adulta. ${ }^{37}$

\section{Considerações finais}

Através dos resultados encontrados, podemos verificar que o perfil antropométrico dos adolescentes estudados revelou excesso de peso, como também de gordura corporal, com resultados acima dos encontrados em outras populações brasileiras estudadas. O perfil cardiorrespiratório mostrou-se consistente com a literatura estudada, com percentual mais elevado para estudantes do sexo masculino. Cabe considerar que as características do perfil bioquímico dos estudantes ingressantes no primeiro ano do segundo grau do IFF-SVS apresentaram resultados inadequados para esta faixa etária, como o verificado nos níveis de colesterol limítrofe e aumentado.

Diante do excesso de peso, gordura corporal e níveis de colesterol total acima do preconizado para a faixa etária, torna-se importante implementar ações de promoção da saúde. Por se tratar de fatores passíveis de intervenção, estratégias de caráter educativo, como medidas de prevenção do aumento de peso, ou manutenção de peso considerados saudáveis, são formas de promover a saúde e qualidade de vida desses alunos.

Por fim, cabe enfatizar a necessidade de ultrapassar a fase diagnóstica e propor alternativas de intervenção, sobretudo quando consideramos que a escola é a instituição responsável pela educação formal e pela difusão de comportamentos saudáveis junto a essa população.

\section{Referências}

1. Eisenstein E, Coelho KSC, Coelho SC, Cruz MAS. Nutrição na adolescência. J. Pediatr. 2000; 76(Supl. 3):S263-S274.

2. Rug PG. Procesos metabólicos y endocrinológicos en la adolescencia. Medicine 2010; 10(61):4196-4201.

3. Traverso-Yépez MA, Pinheiro VS. Adolescência, saúde e contexto social: esclarecendo práticas. Psicol. Soc. 2002; 14(2):33-147.

4. Camargo EAI, Ferrari RAP. Adolescentes: conhecimentos sobre sexualidade antes e após a participação em oficinas de prevenção. Ciên. Saúde Coletiva 2009; 14(3):937-946.

5. Scaduto AA, Barbieri V. O discurso sobre a adesão de adolescentes ao tratamento da dependência química em uma instituição de saúde pública. Ciên. Saúde Coletiva 2009; 14(2):605-614.

6. Brêtas JRS, Ohara CVS, Jardim DP, Muroya RL. Conhecimento sobre DST/AIDS por estudantes adolescentes. R. Esc. Enferm. USP 2009; 43(3):551-557.

7. Garbin CAS, Garbin AJI. A saúde na percepção do adolescente. Physis: R. Saúde Coletiva 2009; 19(1):227-238. 
8. Priore SE, Oliveira RMS, Faria ER, France SC. Nutrição e saúde na adolescência. Rio de Janeiro: Rubio; 2010.

9. Anderson PM, Butcher KF. Reading, writing and refreshments: are school finances contributing to children's obesity? J. Human Resources 2006; 41(3):467-494.

10. Dilley KJ, Martin LA, Sullivan C, Seshadri R Binns HJ. Pediatric Practice Research Group. Identification of overweight status is associated with higher rates of screening for comorbidities of overweight in pediatric primary care practice. Pediatrics 2007; 119(1):148-155.

11. Singh AS, Mulder C, Twisk JW, van Mechelen W, Chinapaw MJ. Tracking of childhood overweight into adulthood: a systematic review of the literature. Obes. Rev. 2008; 9(5):474-488.

12. World Health Organization. Obesity: preventing and managing the global epidemic. Report of a WHO Consultation. Geneva; WHO; 2008. WHO Technical Report Series 894.

13. Perguer R, Melo ME, Halpern A, Mancini MC; Liga de obesidade infantil. Is a diagnosis of metabolic syndrome applicable to children? J. Pediatr. 2010; 86(2):101-108.

14. Heyward VH. Avaliação física e prescrição de exercícios: técnicas avançadas. Porto Alegre: Artmed; 2004.

15. de Onis M, Onyango AW, Borghi E, Siyam A, Nishida C, Siekmann J. Development of a WHO growth reference for school-aged children and adolescents. Bulletin of the World Health Organization 2007; 85:660-667.

16. World Health Organization. Physical status: the use and interpretation of antrometry: report of a WHO expert committe. Genebra: WHO; 1995. WHO Technical Report Series.

17. Taylor RW, Jones IE, Williams SM, Goulding A. Evaluation of waist circumference, waist-to-hip ratio, and the conicity index as screening tools for high trunk fat mass, as measured by dual-energy X-ray absorptiometry, in children aged 3-19 y. Am. J. Clin. Nutr. 2000; 72(2):490-495.

18. Frisancho AR. Anthropometric standards for the assessment of growth and nutritional atatus. Ann Arbor: The University of Michigan Press; 1990.

19. Lohman TG. The use of skinfold to estimate body fatness on children and youth. JOPERD 1987; 58(9): 98-102.

20. Cooper KH. A menos of assessing maximal oxygen intake: correlation between field and treadmill testing. JAMA 1968; 203:135-138.

21. Costa ECC, Guerra LMM, Guerra FEF, Pontes Júniior FL. Validade da medida do consumo máximo de oxigênio e prescrição de intensidade de treinamento aeróbico preditos pelo teste de Cooper de 12 minutos em jovens sedentários. Revista Brasileira de Prescrição e Fisiologia do Exercício 2007; 1(4)32-39.

22. Maior AS, Lima LGM. Respostas agudas hemodinâmicas relacionadas ao Teste de Cooper em militares. Revista da SOCERJ 2008; 21(2)80-87.

23. Sociedade Brasileira de Diabetes. Tratamento e acompanhamento do diabetes mellitus: Diretrizes da Sociedade Brasileira de Diabetes 2007. [acesso em: 21 set. 2011. Disponível em: http://www.anad. org.br/profissionais/images/diretrizes_SBD_2007.pdf

24. Sociedade Brasileira de Cardiologia. I Diretriz de prevenção da aterosclerose na infância e na adolescência. Arquivos Brasileiros de Cardiologia 2005; 85(Supl. 6):1-36. 
25. Beck CC, Lopes AS, Pitanga FJG. Indicadores antropométricos de sobrepeso e obesidade como preditores de alterações lipídicas em adolescentes. Rev. Paul. Pediatr. 2011; 29(1):46-53.

26. Reuter EM, Reuter CP, Burgos LT, Reckziegel MB, Nedel FB, Albuquerque IM, et al. Obesidade e hipertensão arterial em escolares de Santa Cruz do Sul - RS, Brasil. Rev. Assoc. Med. Bras. 2012; 58(6):666-672.

27. Silva RJS, Petroski EL. Consumo máximo de oxigênio e estágio de maturação sexual de crianças e adolescentes. Motricidade 2008; 4(1):13-20.

28. Williams DP, Going SB, Lohman TG, Harsha DW, Srinivasan SR, Webber LS, et al. Body fatness and risk for elevated blood pressure, total cholesterol and serum lipoprotein ratios in children and adolescents. Am. J. Public Health 1992; 82(3):358-363.

29. Dietz WH. Health consequences of obesity in youth: childhood predictors of adult disease. Pediatrics 1998; 101(Supl. 2):518-525.

30. Pereira A, Guedes AD, Verreschi ITN, Santos RD, Martinez TLR. A obesidade e sua associação com os demais fatores de risco cardiovascular em escolares de Itapetininga, Brasil. Arq. Bras. Cardiol. 2009; 93(3):253-260.

31. Instituto Brasileiro de Geografia e Estatística. Pesquisa de orçamentos familiares 2008-2009: despesas, rendimentos e condições de vida [Internet]. Rio de Janeiro: IBGE; 2010. Disponível em: http://www. ibge.gov.br/home/estatistica/populacao/condicaodevida/pof/2008_2009/default.shtm

32. Pereira PB, Arruda IKG, Cavalcanti AMTS, Diniz AS. Perfil lipídico em escolares de Recife - PE. Arq. Bras. Cardiol. 2010; 95(5):606-613.

33. Ribas AS, Silva LCS. Dislipidemia em escolares na rede privada de Belém. Arq. Bras. Cardiol. 2009; 92(6):446-451.

34. Malcom GT, Oalmann MC, Strong JP. Risk factors for atherosclerosis in young subjects: the PDAY Study. Pathobiological determinants of atherosclerosis in youth. Ann. N. Y. Acad. Sci. 1997; 817:179-188.

35. Berenson GS, Srinivasan SR, Bao W, Newman WP, Tracy RE, et al. Association between multiple cardiovascular risk factors and atherosclerosis in children and young adults. N. Engl. J. Med. 1998; 338:1650-1656.

36. Nakashima Y, Fujii H, Sumiyoshi S, Wight TN, Sueishi K. Early human atherosclerosis: accumulation of lipid and proteoglycans in intimal thickenings followed by macrophage infiltration. Arterioscler. Thromb. Vasc. Biol. 2007; 27(5):1159-1165.

37. Porkka KV, Viikari JS, Taimela S, Dahl M, Akerblom HK. Tracking and predictiveness of serum lipid and lipoprotein measurements in childhood: 12-year follow-up: the Cardiovascular Risk in Young Finns Study. Am. J. Epidemiol. 1994; 140(12):1096-1110.

Recebido: $17 / 1 / 2014$

Revisado: 19/12/2014

Aprovado: $14 / 1 / 2015$ 